\title{
Informações nutricionais: o que se tem no país?
}

\author{
Nutritional data: \\ what is available in Brazil?
}

Maria do Carmo Leal 1

Sonia Azevedo Bittencourt 1

\footnotetext{
1 Departamento de Epidemi ol ogia e Métodos Quantitativos em Saúde, Escola Nacional de Saúde Pública, Fundação Oswaldo Cruz.

Rua Leopoldo Bulhões 1480, 8 o andar, Rio de Janeiro, RJ 21041-210, Brasil.
}

Abstract Availablenutritional information in Brazil, generated by non-periodic population surveys, has scarcely contributed to the devel opment of policies aimed at improving the nutritional status of the Brazilian population, although it has hel ped establish the magnitude and distribution of this problem. We suggest that data available in the various health information systems be used to feed, monitor, and evaluate nutritional policies, for updating and local follow-up purposes. Deficiencies in the quality of data available in these health information systems can be minimized through validation studies, thus improving the information generated by the system as a whole and consequently the quality of health care.

Key words Nutrition; Health Information System; Nutritional Surveillance System

Resumo As informações nutricionais existentes no País, oriundas de inquéritos populacionais sem periodici dade definida, embora contribuindo na defini ção da magnitude e distribui ção do problema, têm se mostrado de pouca utili dade para subsi diar a formulação de políticas que redundem na mel horia do estado nutricional da população brasileira. Sugere-se consi derar os dados di sponívei s nos di versos sistemas rotineiros de informação da área de saúde para alimentar, monitorar e avaliar as políticas nutricionais, possi bilitando atuali dade e acompanhamento local. As deficiências na qualidade dos dados nesses sistemas da área da saúde podem ser minimizadas através de estu dos de validação, gerando uma mel horia das informações do sistema como um todo, com reflexos na qualidade da assistência à saúde.

Palavras-chave Nutrição; Sistema delnformação em Saúde; Sistema deVigilância Nutricional 
O estado nutricional de uma população é um indicador positivo de saúde, ao contrário da maioria dos indicadores clássicos de morbidade e mortalidade, que mede a ausência de saúde. A nutrição humana é uma das áreas mais importantes do ponto de vista da saúde e do bem-estar das populações. Os problemas nutricionais do povo brasileiro são diversos, expressando-se de modo diferenciado nos vários grupos sociais. Em um extremo, observa-se a carência alimentar, eticamente uma das mais elementares e chocantes privações do mundo atual. Em outro, a obesidade, que provavelmente constitui, de forma isolada, o maior fator de risco biológico para o adoecimento na idade adulta, contribuindo, assim, para onerar enormemente os sistemas de saúde. A convivência desses extremos traz grandes desafios ao setor saúde na execução de políticas que contemplem necessidades tão distintas.

A maioria dos países, principalmente os de maior desenvolvimento sócio-econômico, investiu na implementação de sistemas de informação nutricional das suas referidas populações. Em 1974, realizou-se, em Roma, a Conferência Mundial de Alimentação, sob a coordenação da Food and Agriculture Organization (FAO), cuja principal recomendação foi que os países assegurassem o acesso aos alimentos a toda a população humana. A partir daí, verificou-se uma maior ênfase na construção de sistemas de informação que pudessem monitorar a situação alimentar e nutricional (FAO, 1974).

No setor saúde, entende-se a Vigilância Nutricional como uma ferramenta essencial para a tomada de decisões. Esta, portanto, deve abranger informações geradas a partir tanto de estudos populacionais desenhados para esta finalidade, quanto da coleta sistemática e contínua de informações dos serviços de saúde.

Existe, no Brasil, uma experiência acumulada na realização de inquéritos nutricionais, o que tem permitido conhecer a magnitude do problema em alguns períodos e, mesmo, ter uma noção evolutiva, com base na comparabilidade desses resultados.

O primeiro inquérito nutricional com dimensão nacional foi realizado em 1974/ 1975, sob a coordenação do Instituto Brasileiro de Geografia e Estatística (IBGE) e assessoria da FAO - Estudo Nacional de Despesa Familiar (ENDEF) - , e teve como objetivo principal avaliar o consumo alimentar e a estrutura de despesa familiar, além do estado nutricional de uma amostra da população brasileira representativa em nível dos Estados da Federação (Viacava et al., 1983). Foram avaliadas com dados antropométricos 55 mil famílias no País, enquanto em uma subamostra, para avaliar o consumo alimentar, utilizou-se a metodologia de pesagem direta de alimentos pelo período de uma semana. Embora esse estudo tenha sido de inegável importância para o conhecimento do estado nutricional e do consumo de alimentos, seus resultados só puderam ser conhecidos dez anos após a sua realização.

Em 1987/ 1988, um outro inquérito, também sob a coordenação do IBGE, foi realizado nas regiões metropolitanas e nas ci dades de Brasília e Goiânia: a Pesquisa de Orçamentos Familiares (POF). Esse estudo investigou 13.611 domicílios, abordando apenas os itens das despesas familiares, não se detendo na tomada de medidas antropométricas. Ainda assim, pôdese determinar, de forma indireta, o consumo de alimentos das famílias nas áreas estudadas (Bittencourt et al., 1992).

Em 1989, foi realizada a Pesquisa Nacional sobre Saúde e Nutrição (PNSN), com o objetivo principal de avaliar o estado nutricional da população brasileira, além de caracterizar as condições de saúde e estrutura sócio-econômica das famílias investigadas. Foram estudadas 14 mil famílias distribuídas por todo o território nacional, com representatividade macrorregional e urbano-rural (INAN, 1990).

Mais recentemente, na década de 90 , o Instituto Nacional de Alimentação e Nutrição (INAN), em conjunto com universidades brasileiras, realizou um estudo multicêntrico com o intuito de avaliar a nutrição, o consumo alimentar e a atividade física de uma amostra de indivíduos residentes em sete cidades brasileiras, a saber: Rio de Janeiro, Curitiba, Goiânia, São Paulo, Salvador, Campinas e Ouro Preto. No total foram avaliados cerca de mil domicílios por cidade (INAN/MS, 1997).

$\mathrm{Na}$ área da saúde materno-infantil, os dados mais atuais referem-se à Pesquisa Nacional sobre Demografia e Saúde, realizada pela Sociedade Civil Bem-Estar Familiar no Brasil (BENFAM ). Foram entrevistados os residentes de 13.283 domicílios, onde, entre outras informações, foi avaliado o estado nutricional de crianças menores de cinco anos e o das mães. A amostra da pesquisa foi desenhada para obter resultados representativos para o Rio de Janeiro, São Paulo, Minas Gerais, Rio Grande do Norte, Bahia, Pernambuco, Ceará e Rio Grande do Sul, bem como para as Regiões Sul, CentroLeste, Nordeste, Norte e Centro-Oeste (BENFAM, 1997).

Outros estudos, de abordagem estadual, regional ou, mesmo, municipal, têm sido conduzidos nos últimos anos, como, por exemplo, o Censo de Altura de Escolares, realizado nos Es- 
tados do Ceará, Paraíba e Piauí (SEE/CE et al., 1993; SEE/PB et al., 1993; SEE/PI et al., 1993); há também aqueles realizados em populações definidas, sobretudo com crianças, tais como o estudo longitudinal de Pelotas (Victora et al., 1995) e a pesquisa sobre saúde e nutrição das crianças de São Paulo (Monteiro, 1988), entre outros. Além disso, estudos específicos sobre patologias relacionadas com a questão nutricional têm gerado informações importantes para o conhecimento e atualização do problema, como é o caso do Estudo Multicêntrico sobre a Prevalência de Diabetes Mellitus, envolvendo adultos de 30 a 69 anos de nove capitais brasileiras, realizado entre 1986 e 1988 (CCCEM PDM , 1992). Também têm trazido contribuições os estudos sobre carências específicas, particularmente os dois inquéritos nacionais sobre bócio endêmico, realizados entre escolares nos anos de 1955 e 1975 (Batista Filho \& Barbosa, 1985). Em menor escala, mas não menos importantes, estão os inquéritos de prevalência de anemia ferroprívica e de hipovitaminose A realizados em áreas sob risco.

Todo esse esforço foi fundamental para compreender a magnitude, distribuição geográfica e fatores de risco associados, bem como para destacar a relevância do problema nutricional no Brasil. Porém, o alto custo, a demora na disponibilização dos resultados, a falta de representatividade em nível local e a intermitência dessas informações têm-nas tornado de pouca utilidade prática para subsidiar a formulação de políticas que redundem na melhoria do estado nutricional da população brasileira. Pode-se mesmo observar que, em comparação com a experiência internacional, o Brasil encontra-se em um estágio inicial de desenvolvimento de um sistema de informação sobre nutrição (Mock \& Bertrand, 1993). A opção por inquéritos ocasionais reafirma a ausência de um sistema de informação contínuo.

Na tentativa de suprir importantes lacunas do conhecimento sobre o estado nutricional da população brasileira, em 1990, foi instituído nos serviços de saúde, pelo Ministério da Saúde, sob a responsabilidade do INAN, o Sistema deVigilância Alimentar e Nutricional (SISVAN) (INAN/ MS, 1996). Mais recentemente, a expansão do SISVAN está associada ao Programa de Atendimento aos Desnutridos e à Gestante em Risco Nutricional. Apesar dos esforços, esse sistema de informação é pouco representativo dos serviços básicos de saúde, além de o mesmo se propor a cobrir apenas os serviços públicos e se restringir à população de gestantes e crianças.

Embora com limitações, os sistemas de informações instituídos da área da saúde podem contribuir para o monitoramento da situação nutricional.

O indicador peso ao nascer dos nascidos vivos pode refletir, em grande parte, a nutrição materno-fetal e a probabilidade de sobrevivência da criança no primeiro ano de vida. Universalmente utilizado com esta finalidade, pode atual mente ser conhecido na maioria dos municípios brasileiros através do Sistema de Informação sobre Nascidos Vivos (SINASC) (Mello Jorge et al., 1996). Sendo um sistema descentralizado, de responsabilidade municipal, há a possibilidade de ser coletado, processado e analisado em tempo hábil, a fim de permitir uma utilização oportuna.

Indicadores de mortalidade obtidos do Sistema de Informação sobre Mortalidade (SIM) podem contribuir, sobretudo, para o monitoramento dos resultados nefastos e indesejáveis da ausência ou precariedade de uma política pública sobre nutrição humana. Em relação às crianças (principalmente as menores de cinco anos), de uma maneira direta podem-se acompanhar os óbitos ocorridos por desnutrição, tanto como causa básica de óbito, quanto como causa associada. Tendo-se conhecimento sobre a associação das diarréias e pneumonias com a desnutrição infantil, podem-se também utilizar essas causas como indicadores do mesmo problema (Carvalho et al., 1990). Em relação aos adultos, os problemas nutricionais mais freqüentes são oriundos da obesidade, que se caracteriza como fator de risco importante para várias patologias, como as doenças cardiovasculares e cânceres, além das doenças metabólicas (destaque para o diabetes melittus). Nos idosos, segmento da população que mais cresce em todo o mundo, pode-se considerar o retorno de alguns problemas da infância (desnutrição, diarréias e infecções), além das patologias acumuladas no percurso da vida adulta.

Um outro sistema é o de Informação Hospitalar (SIH), que é pouco utilizado, de confiabilidade menor, mas muito promissor. Refere-se ao conjunto de atendimentos ocorridos devido a patologias graves, que demandaram hospitalização. Pode cobrir um outro conjunto de doenças que não se representam bem nas estatísticas de mortalidade, como, por exemplo, a hipertensão arterial, osteoartrites, cálculos renais, patologias associadas com a obesidade. Esse sistema de informação seria particularmente interessante para a avaliação de gastose custos com o adoecimento relacionado direta e indiretamente à questão nutricional.

Menos organizado, mas com possibidades de grande contribuição, é o Sistema de Geren- 
ciamento de Unidade Ambulatorial Básica (SI$\mathrm{GAB}$ ), que pode cobrir um elenco mais amplo de patologias clínicas com manifestação leve que não estariam representadas nos sistemas anteriormente descritos, como, por exemplo, a desnutrição moderada e a hipertensão arterial leve. A intervenção neste nível evitaria o agravamento da maioria dos problemas de saúde de base nutricional.

Há que se destacar a necessidade de estudos de validação que permitam corrigir as distorções dos dados dos sistemas oficiais. Certamente com custos muito inferiores aos inquéritos populacionais, tais estudos seriam de grande utilidade para o melhor monitoramento dos problemas nutricionais e implementariam a qualidade da informação, beneficiando, simultaneamente, a execução e a avaliação de outros programas de saúde.

Esses dados advindos dos sistemas rotineiros de informação são importantes para o acompanhamento das patologias relacionadas aos problemas nutricionais, sobretudo no contexto político atual da descentralização, quando a representatividade de dados em nível local é uma necessidade.

A utilização das informações geradas pelos sistemas de informações da área da saúde não elimina a obrigatoriedade de o País realizar inquéritos periódicos de saúde, que viriam não somente completar as informações de morbidade existentes, mas também validá-las. Ade- mais, eles permitiriam conhecer o perfil antropométrico e bioquímico dos nutrientes na população brasileira, a exemplo do que ocorre em alguns países. Os inquéritos periódicos de saúde constituem a principal fonte de dados sobre nutrição nos países que os realizam rotineiramente. Um outro banco de dados utilizado refere-se à inscrição no Serviço Militar, o que, devido à sua obrigatoriedade, corresponde a um censo anual sobre as condições antropométricas e de saúde da população masculina de 18 anos de idade.

A não-utilização das informações atualmente disponíveis, que proporcionariam uma visão parcial do problema, na verdade reflete o descaso quanto ao desenvolvimento de uma política nutricional para o Brasil, a qual certamente exigiria parcerias intersetoriais, extrapolando, assim, o âmbito da saúde. Nesse sentido, existe a necessi dade de efetivar um organismo multissetorial que congregue, pelo menos, os Ministérios da Saúde, Agricultura, Educação e Economia, com a finalidade permanente de definição de estratégias, ações e avaliação da situação alimentar e nutricional da população.

A ausência de um monitoramento do estado nutricional da população brasileira não condiz com o estágio de desenvolvimento econômico alcançado pelo País, que necessitaria de um sistema de informação ágil e de qualidade para estar sistematicamente revendo suas diretrizes na política de saúde e nutrição.

\section{Referências}

BATISTA FILHO, M. \& BARBOSA, N., 1985. Alimentação e Nutrição no Brasil, 1974-1985. Pró-Memória. Brasília: Canadá Gráfica.

BENFAM (Sociedade Civil Bem-Estar Familiar no Brasil), 1997. Pesquisa Nacional sobre Demografia eSaúde 1996. Rio de Janeiro: BENFAM.

BITTENCOURT, S. A.; BOSI, M. L. M.; OLIVEIRA, E. S.; SILVA, D. O.; MENEZES, F. A. \& MEDICI, A., 1992. Nutrição, meio ambiente e desenvolvimento: contribuição à Conferência Mundial de Ecologia e Meio Ambiente. In: Saúde, Ambientee Desenvolvimento (M. C. Leal, org.), vol. 2, pp.123-151, Rio de Janeiro: Abrasco/São Paulo: Hucitec.

CARVALHO, M. L.; NIOBEY, F. M. L.; MIRANDA, N. N. \& SABROZA, P. C., 1990. Concordância na determinação da causa de óbito nos menores de um ano na região metropolitana do Rio de Janeiro. Revista deSaúde Pública, 24:20-27. 
CCCEMPDM (Comissão Coordenadora Central do Estudo Multicêntrico sobre Prevalência do Diabetes Mellitus), 1992. Estudo multicêntrico sobre a prevalência do diabetes mellitus. Informe Epidemiológico do SUS, 3:47-73.

FAO (Food and Agriculture Organization), 1974. Conferencia Mundial delos Alimentos. Roma: FAO.

INAN (Instituto Nacional de Alimentação e Nutrição), 1990. Pesquisa Nacional sobre Saúde e Nutrição. Resultados Preliminares. 2a ed., Brasília: INAN, Ministério da Saúde.

INAN (Instituto Nacional de Alimentação e Nutrição), 1996. Manual deInstrução sobre o Fluxo de Informação da Vigilância Nutricional. Brasília: INAN, Ministério da Saúde.

INAN (Instituto Nacional de Alimentação e Nutrição), 1997. Relatório Preliminar do Estudo Multicêntrico sobre Consumo Alimentar. Campinas: INAN, Ministério da Saúde.

MELLO JORGE, M. H.; GOTILIEB, S. L. D.\& OLIVEIRA, H., 1996. O sistema de informação sobre nascidos vivos: primeira avaliação dos dados brasileiros. Informe Epidemiológico do SUS, 2:15-48.

MOCK, N. B. \& BERTRAND, W. E., 1993. Marco conceptual para los sistemas de vigilancia nutricional. Boletin dela Oficina Sanitaria Panamericana, 115:511-522.
MONTEIRO, C. A., 1988. Saúde Nutrição das Crianças deSão Paulo. São Paulo: Hucitec/EDUSP.

SEE/CE (Secretaria de Estado de Educação do Ceará); FAE (Fundação de Assistência ao Estudante) \& PMA (Programa Mundial de Alimentos), 1993. Primeiro Censo Estadual deAltura dos Escolares da Primeira Série do Ensino Fundamental do Estado do Ceará. Fortaleza: SEE/CE.

SEE/ PB (Secretaria de Estado de Educação da Paraíba); FAE (Fundação de Assistência ao Estudante) \& PMA (Programa Mundial de Alimentos), 1993. Avaliação do Estado Nutricional de Crianças em Idade Escolar no Estado da Paraíba (Censo deAltura). João Pessoa: SEE/PB.

SEE/PI (Secretaria de Estado de Educação do Piauí); FAE (Fundação de Assistência ao Estudante) \& PMA (Programa Mundial de Alimentos), 1993. Primeiro Censo Estadual de Altura em Escolares da Primeira Série do Ensino Fundamental do Estado do Piauí. Teresina: SEE/PI.

VIACAVA, F.; FIGUEIREDO, C. M. P. \& OLIVEIRA, W., 1983. A Desnutrição no Brasil: Uma análise do Estudo Nacional de Despesa Familiar para o Nordeste, Estado de São Paulo e Estado do Rio de Janeiro. Brasília: Vozes.

VICTORA, C. G.; BARROS, F. C. \& VAUGHAN, J. P., 1995. Epidemiologia da Desigualdade: Um Estudo Longitudinal de 6.000 Crianças Brasileiras. São Paulo: Cebes/Hucitec. 